\title{
IL TEMA DELLA VIOLENZA IN BUIO, DI DACIA MARAINI
}

\author{
Tanja Habrle \\ Martina Popović \\ Università Juraj Dobrila di Pola \\ Dipartimento di studi in lingua italiana
}

\section{Riassunto}

Il saggio offre una breve analisi di alcuni racconti, ispirati a fatti realmente accaduti, contenuti nella raccolta Buio, pubblicata da Dacia Maraini, per i tipi delleditore Rizzoli, nel 1999 e risultata vincitrice, in quello stesso anno, del Premio Strega. In questa analisi l'attenzione sarà rivolta alle principali tematiche care all'autrice, ed in particolar modo a quella della violenza sui bambini. La prospettiva che quest'opera ci offre è infatti popolata da bambini violentati, venduti e uccisi, ma soprattutto circondati da una profonda indifferenza della società verso tali devastanti violenze. Si è cercato pertanto di elaborare, attraverso l'analisi dei racconti di Dacia Maraini, un percorso che porti ad una interpretazione che induca a riflettere sulle dure tematiche trattate dall'autrice, al fine di promuovere la speranza per le vittime di violenze ed abusi, nella convinzione che un mondo libero da qualsiasi tipo di violenza sia possibile.

Parole chiave: Dacia Maraini, Buio, letteratura italiana, infanzia, violenza.

L'esperienza vissuta nel lager nazista in Giappone, i bombardamenti, la fame, il freddo, hanno portato Dacia Maraini ${ }^{[1]}$ a farsi portavoce dei più deboli ${ }^{[2]}$. Il suo

\footnotetext{
[1] Dacia Maraini è nata a Fiesole (Firenze) il 13 novembre del 1936. La madre, Topazia Alliata, fu pittrice ed appartenne allantica famiglia siciliana degli Alliata di Salaparuta, mentre il padre Fosco Maraini, per metà inglese e per metà fiorentino, fu un famoso etnologo ed autore di numerosi saggi e libri fotografici sul Tibet e sull'Estremo Oriente. La scrittrice Dacia Maraini studiò a Palermo e compiuti i diciotto anni e in seguito alla separazione dei genitori, si trasferì con il padre a Roma, dove per guadagnare si adattò a diversi lavori precari di archivista, aiuto fotografa, segretaria e giornalista saltuaria. A soli ventun anni la Maraini fonda, con Marisa Gambardella e Angela Giannitrapani, la rivista Tempo di letteratura, pubblicata dalleditore Pironti di Napoli, che però pretese che il ruolo di Direttore fosse coperto da un uomo (è per questo motivo che a firmarla fu il marito di Angela Giannitrapani, Nino Minissi). Nello stesso periodo inizia la collaborazione dellautrice con diverse riviste come Paragone, Nuovi Argomenti e Il Mondo. Nel 1962 viene pubblicato il primo romanzo della Maraini, intitolato La vacanza, che sarà nuovamente pubblicato da Bompiani nel 1976 e poi da Einaudi nel 1988. Nel 1967 la scrittrice fonda il Teatro del Porcospino, con la collaborazione di Enzo Siciliano, Lorenzo Tornabuoni, Carlotta Barilli, Paolo Bonacelli, Carlo Montagna e Roberto Guicciardini. Nel 1973 la Maraini fonda, con la collaborazione di Lù Leone, Francesca Pansa e Marida Boggio il Teatro della Maddalena, gestito e diretto esclusivamente da donne.

[2] Nel 1938 il lavoro del padre portò la famiglia Maraini in Giappone, in quanto Fosco Maraini vinse una borsa di studio per condurre ricerche sugli Hainu, una popolazione giapponese in via d'estinzione che viveva nell'isola di Hokkaido. Nel 1943 il governo giapponese, in base al patto d’alleanza stipulato con l'Italia e la Germania, chiese ai coniugi Maraini di firmare l'adesione alla Repubblica di Salò. I
} 
impegno femminista l'ha condotta ad usare la scrittura per presentarci un ritratto della società contemporanea, caratterizzata da ipocrisie e violenze. Con uno sguardo da sempre rivolto alla giustizia, con coerente impegno nel denunciare i mali della società, la scrittrice ha arrecato, con le proprie opere, ${ }^{[3]}$ un rilevante contributo al dibattito sulla violenza ai danni di donne e bambini. È infatti principalmente ai più deboli, agli emarginati, alle donne e ai bambini, che la nostra autrice si propone, attraverso la propria creazione artistica, di dar voce. Nella sua raccolta intitolata Buio, pubblicata nel 1999 per i tipi di Rizzoli, veniamo per così dire immersi in una realtà oscura, in cui gli orrori diventano reali e le paure prendono corpo e forma. Dodici storie che ci riguardano, dodici protagonisti, tra cui bambini, donne ed adolescenti maltrattati, che vedono attorno a sé l'indifferenza del mondo. Attraverso le orrende vicende narrate in ciascuna delle storie, ci accompagna, come in un giallo, la guida sicura di una commissaria di polizia, Adele Sòfia, intenta a cercarne i colpevoli allo scopo di assicurarli alla giustizia.

Il tema della violenza esercitata sui bambini non è nuovo. Basta sfogliare opere quali la Storia dell'educazione di Egle Becchi (La Nuova Italia, 1987), Innocenza violata di Caterina Fischetti (Franco Angeli, 1996) e la Storia dell'infanzia di Lloyd De Mause (Emme, 1983) per venire a conoscenza del fatto che la violenza sui bambini affonda le proprie radici (ed è documentata) già nell'antichità.

"La storia dell'infanzia è un incubo dal quale solo di recente abbiamo cominciato a destarci. Più si va addietro nella storia, più basso appare il grado di attenzione per il bambino, e più frequentemente tocca a costui la sorte di venire assassinato, abbandonato, picchiato, terrorizzato, e di subire violenze sessuali."[4]

Nel passato, solo per fare un esempio, in Inghilterra i figli indesiderati (ed in particolar modo le figlie), come pure i bambini con difetti psicologici o fisici, venivano strangolati, oppure gettati nel Tamigi, ed era culturalmente accettato che le punizioni venissero inflitte con la frusta o mettendo i bambini a pane ed acqua.

"Era solito usare la frusta con freddezza, lentamente e deliberatamente. Chiedeva al ragazzo di calare i calzoni, e di allungarsi sul banco, e ci dava dentro con le cinghie di cuoio. "In ognuno dimora uno Spirito del Bene e uno Spirito del Male. Lo Spirito del Bene ha il suo

due, essendo antifascisti, naturalmente rifiutarono di firmare e vennero perciò internati, con le tre figlie, in un campo di concentramento a Tokyo, dove per due anni patirono la fame, il freddo, i bombardamenti e furono liberati dagli americani solo dopo la fine della guerra (delle atroci sofferenze vissute in quegli anni, Dacia Maraini scriverà nella raccolta di poesie intitolata Mangiami pure, del 1978, e nell’opera Buio, pubblicata nel 1999).

[3] Numerose sono le opere in cui Dacia Maraini pone l'accento sulle diverse forme di violenze, alcune delle più importanti opere sono: La lunga vita di Marianna Ucrìa (Rizzoli Editore, 1990), Buio (Rizzoli Editore, 1999), L'età del malessere (Einaudi Editore, 1963), Voci (Rizzoli Editore, 1994), L’amore rubato (Rizzoli Editore, 2012).

[4] De Mause, L. (a cura di), Storia dell'infanzia, Milano, Emme Edizioni, 1983, pag. 9. 
domicilio nella testa; anche lo Spirito del Male ce l'ha, ed è il posto dove ricevi le frustate.»" ${ }^{\text {[5] }}$

Nonostante i mass media forniscano con troppa frequenza notizie di fatti di cronaca riguardanti violenze su bambini, tali notizie vengono presto dimenticate nella fretta della vita quotidiana. Dacia Maraini ci porge invece, con i suoi racconti, una testimonianza scritta durevole, con la quale intende risvegliare la società e rompere il silenzio, per contribuire a creare un futuro in cui i bambini non debbano più conoscere le devastazioni causate dalla violenza.

La raccolta si intitola metaforicamente Buio, per alludere a quella che è probabilmente la maggiore fonte di paura per ogni bambino ${ }^{[6]}$, ma certo anche per riferirsi a ciò che il buio rappresenta pure nell'immaginario collettivo degli adulti, vale a dire ciò che è oscuro, occulto, pericoloso, e quindi con riferimento alla pesantezza delle storie realmente accadute e raccontate dalla scrittrice.

Il racconto con cui la raccolta Buio si apre presenta la storia di un bambino, Grammofono, che deve ancor compiere sette anni e che passa le giornate in casa, da solo, perché i genitori lavorano. La Maraini, in questo racconto, non fornisce nessuna informazione sui genitori, ma si concentra su come il bambino trascorre le mattinate giocando e, soprattutto, osservando i piccioni, che rappresentano il suo principale oggetto d'interesse. Una mattina, proprio mentre il bambino sta osservando i piccioni, un uomo, seduto sulla panchina nei giardini di fronte alledificio in cui Grammofono vive, inizia a buttare sassolini per catturare l'attenzione del bambino. Lunica regola che i genitori del bambino gli avevano imposto era di non uscire di casa, ma la curiosità era troppo forte ed un giorno Grammofono decide di andare a conoscere quest'uomo, disobbedendo ai genitori.

"L'uomo piccione prende per mano il bambino Grammofono e lo conduce verso una automobile parcheggiata all'angolo della via. [...] L'uomo ora ha fermato la macchina fra due cabine dei lavori stradali, sprangate. Non c’è nessuno in giro fra quei pini. Le mani pennute si allungano verso il bambino; gli allargano il collo della maglietta, gli slacciano la cintura dei pantaloncini. [...] L'uomo ora gli è addosso. Lo tiene stretto. Gli urla nell'orecchio «se continui a tirare calci ti ammazzo». Il bambino gli dà una ginocchiata nel ventre. L'uomo urla di dolore. Prende il bambino per il collo e stringe rabbiosamente le dita sulla giugulare. [...] La mattina dopo lo spazzino trova il cadavere di un bambino mezzo nudo, con le

[5] Levin, S., Childhood in exile, Harcourt Brace, New York, 1929, pp. 58-59.

[6] Sigmund Freud descrive la percezione che i bambini hanno del buio: "Hanno paura del buio perché nel buio non possono vedere la persona che amano; e la loro paura si attenua se nel buio possono tenere la mano di tale persona." In Freud, S., Aforismi e Pensieri, Aonia edizioni, Pisa, 2012, pag. 46. 
scarpe da ginnastica rosse ai piedi, nella pineta sopra la città.,'[7]

Il racconto prosegue descrivendoci passo per passo la ricerca dell'aggressore e la dettagliata indagine portata avanti dalla commissaria Adele Sòfia, il cui unico fine è di trovare l'assassino. La storia del bambino Grammofono, stuprato e ucciso, rappresenta la realtà che ci circonda, non di rado crudele ed orrenda, ed il cui orrore arriva talvolta al proprio culmine proprio quando si viene a conoscere dell'identità degli aggressori. Come avviene appunto in questo caso, in cui Dacia Maraini spiazza il lettore indica come aggressore un assistente sociale, che trascorre il proprio tempo lavorando con bambini affetti da sindrome di Down.

La Maraini dimostra qui la sua bravura, usando uno stile narrativo pacato e semplice per descrivere atti disumani, come lo stupro dei bambini, che nella maggior parte dei casi termina con l'assassinio delle vittime. Un prezzo troppo alto pagato da bambini innocenti, circondati da una società distratta verso tali problemi e che decide di non reagire, lasciando spazio alle menti disturbate degli aggressori che, grazie al disinteresse sociale, rimangono impuniti.

La protagonista del racconto Viollca la bambina albanese è appunto Viollca, una bambina di dodici anni che dall'Albania si imbarca per andare in Italia. L'intento dichiarato dei genitori è quello di allocare la figlia presso dei signori italiani: lei deve andare, guadagnare soldi e poi tornare in Albania dai genitori. Arrivata in Italia, la bambina viene portata in un appartamento dal quale le viene proibito di uscire e dove conosce un'altra bambina albanese, anch'essa venuta in Italia per lavorare. La sera seguente le due bambine vengono lavate e preparate per andare al lavoro

"Con le gonne al sedere, le gambe velate da calze a rete, il reggicalze rosso che sbuca da sotto le mutande, $i$ tacchi alti, il top scintillante e la giacchina di velluto su cui spiccano $i$ riccioloni biondi, le due ragazzine appaiono sulla porta, sbalordite, come due personaggi di fumetti porno."[8]

Vengono condotte in una piazza quadrata, in cui le accoglie una donna, complice dei due trafficanti che ora svolgono la parte dei loro "padroni", che spiega loro in che modo svolgere il proprio lavoro, che consiste nel vendere il proprio corpo a uomini più grandi. Le bambine, confuse, vengono portate in camere separate, in cui devono stare ferme ed aspettare i clienti. A questo punto inizia l'incubo in cui i corpi innocenti di queste bambine vengono sfruttati da diversi uomini, di diverse età. Le due bambine 
vengono violentate più volte nel corso della serata, senza mai vedere i soldi guadagnati.

Al termine del racconto Dacia Maraini inserisce un finale positivo, poiché le due bambine vengono salvate grazie ad un'indagine della polizia guidata dalla commissaria Adele Sòfia; compiuti i diciotto anni le due bambine sarebbero state uccise o vendute ad un altro trafficante.

Nel racconto Alicetta la scrittrice ci introduce nella triste storia di un'amabile bambina, Alicetta che a nove anni è rimasta orfana ed è stata cresciuta grazie alle cure del nonno, affetto da schizofrenia. Non parla, tiene la testa inclinata, ma è molto buona ed intelligente. Il nonno e Alicetta sono riusciti a vivere senza problemi per quasi un anno grazie alla pensione del nonno, che però, dopo essersi ammalato, non riuscendo più a prendersi cura della nipote per qualche mese, cerca aiuto in una clinica.

Le cure di Alicetta vengono assegnate al capoinfermiere e al portantino, che la lavano, la pettinano e le danno da mangiare. La salute della bambina sembra però peggiorare di giorno in giorno, finché una mattina il suo corpo viene trovato senza vita. Ha inizio quindi un' indagine, condotta dalla commissaria Adele Sòfia, mediante la quale si scopre, dopo diversi interrogatori, che i due somministravano alla bambina grosse dosi di sedativi, la portavano ai bagni e poi la violentavano, finché, dopo troppe aggressioni, il piccolo corpo di Alicetta aveva ceduto.

L'orrore di questo racconto raggiunge il culmine quando il lettore viene a conoscenza del fatto che due persone, il cui lavoro dovrebbe consistere nel prendersi cura delle bambine, decidono di abusare della propria posizione per soddisfare i propri bisogni animaleschi, non lasciando via di scampo alle loro vittime.

Nel racconto intitolato Il pastore Ahmed e le tre ragazze nel bosco, guidati di nuovo dall'occhio esperto della commissaria Adele Sòfia, veniamo condotti sulla cima di una montagna per conoscere il pastore Ahmed, un pover'uomo raccattato dalla strada dall'avvocato Tronci, che lo ha messo lassù perché si prenda cura delle sue pecore. Ahmed è abituato a non sentire voci umane, si rilassa ascoltando il fruscio delle foglie e il verso delle cicale, ma ad un tratto un suono sconosciuto lo spaventa e si sente chiudere lo stomaco.

"Il pastore Ahmed inghiotte saliva guardandole avanzare, cosi fresche e innocenti. Più che attraenti però le trova rovinose, arroganti nella loro ignoranza di sé. Come si può permettere a delle ragazze di camminare a quel modo, di raparsi $i$ capelli, di appendere alle 
orecchie dei ciondoli d'argento che penzolano osceni ad ogni moto del capo? Al suo paese una cosa simile non avverrebbe mai. ${ }^{\prime[9]}$

Vedendo la carne nuda delle ragazze ed il modo in cui camminano e saltellano, il pastore prega il suo Dio, prega Allah di non fargli vedere ciò che vede. Come per incanto, il pastore non riesce più a distinguere le figure davanti ai suoi occhi: vede solo ombre che si muovono senza senso. Un paio d’ore più tardi, una delle ragazze scende al bar in città, con i vestiti sciupati, tutta la faccia coperta di sangue e le ginocchia sbucciate; non riesce a dire una parola, e cade infine a terra priva di sensi.

L'unico sospettato è il pastore Ahmed, ma non ci sono prove per incriminarlo; inoltre la commissaria Adele Sòfia, alla quale è stato assegnato il caso, non crede che il pastore sia il colpevole e decide di interrogare il padrone delle pecore, ma anche in questo caso non emergono indizi che possano incriminarlo. A distanza di sei mesi, però, Donatella Lumi, l'unica ragazza sopravvissuta, ricorda tutto quello che è successo: lei e le sue due amiche si sono incamminate verso il monte; qualcuno le ha seguite, poi le ha minacciate con un coltello e legate ad un albero. Donatella è poi riuscita a liberarsi, ha corso più che poteva, ma è arrivata in paese troppo tardi, ed in uno stato di shock che le ha fatto dimenticare tutto l'accaduto. Alla vista dell'avvocato, però, inizia ad urlare, avendolo finalmente riconosciuto. Era vero quindi, Ahmed non aveva visto ciò che non voleva vedere, la sua preghiera gli aveva davvero salvato la vita.

Concludendo, si può dire che la violenza non può essere giustificata. Per secoli troppi bambini hanno subito l'impatto della violenza di adulti che intenzionalmente hanno esercitato il loro potere, malato e maniacale, sulla loro impotenza, fisica e psichica, a difendersene. Non si parla neppure di un problema regionale o statale, perché la violenza sui minori è un tema globale, che assume semmai variegate forme locali, come la mutilazione degli organi genitali femminili, abusi sessuali oppure omicidi. Per molto tempo questi tipi di violenze sono stati mascherati sotto l'etichetta di "tradizioni" o "usi" locali.

Nell'ambito della famiglia, inoltre, la violenza sui bambini viene spesso accompagnata da una profonda paura, per cui i bambini tacciono ed in molti casi, se la violenza viene esercitata dai genitori, i bambini tendono ad esser visti come bugiardi o dotati di eccessiva immaginazione, per cui spesso non vengono presi sul serio. Perciò nelle pagine di Dacia Maraini la violenza sui minori è solitamente accompagnata dal silenzio: si tratta di vittime che non hanno la possibilità di parlare e, se parlano, non

[9] Ivi, pag. 186. 
vengono ascoltate. La scrittrice mette inoltre spesso in risalto il ruolo passivo di una società cieca e muta, in cui l'educazione viene talvolta impartita attraverso punizioni umilianti che, nella maggior parte dei casi, consistono in violenze fisiche e psichiche. La Maraini si sofferma in modo particolare sull'innocenza dei bambini, che, anche volendo, non possono sottrarsi alla violenza degli adulti, essendo da un lato privi della necessaria forza fisica e psichica, e dall'altro totalmente dipendenti dalla cura degli adulti. Attraverso i racconti che ci presenta ne il Buio l'autrice intende indurci a riflettere sulla nostra cultura e sul nostro modo di affrontare la violenza; ora più che mai - sembra volerci dire - bisogna porre fine al silenzio, perché i bambini hanno il bisogno di essere ascoltati, e noi dobbiamo porgere loro una via d'uscita, una speranza, che possa portarli a vedere la violenza come qualcosa di anormale, di inaccettabile. I bambini hanno il diritto di parlare, di denunciare, di avere un'infanzia libera da ogni oppressione, violenza e umiliazione, mentre la società deve sentirsi in obbligo di rispettare questi diritti, facendo di più per prevenire le sofferenze devastanti causate dalla violenza. 


\section{BIBLIOGRAFIA}

CATTARUZZA, C. (a cura di), 2000, Dedica a Dacia Maraini, Lint, Trieste.

CRUCIATA, M. A., 2003, Dacia Maraini: una voce dal "genere", Cadmo, Fiesole.

BECCHI, E. (a cura di), 1987, Storia delleducazione, La Nuova Italia, Firenze.

DE MAUSE, L. (a cura di), 1983, Storia dell'infanzia, Emme Edizioni, Milano.

FISCHETTI, C., 1996, Innocenza violata. Storie, riflessioni, proposte per combattere la violenza sui (ai) minori, Editori Riuniti, Roma.

FREUD, S., 2012, Aforismi e Pensieri, Aonia edizioni, Pisa.

LEVIN, S., 1929, Childhood in exile, Harcourt Brace, New York.

MARAINI, D., 1999, Buio, Rizzoli Editore, Milano.

\section{Sitografia}

www.treccani.it/enciclopedia/dacia-maraini/

www.daciamaraini.com 


\section{NASILJE U DJELU BUIO (TAMA) - DACIA MARAINI}

Ovaj rad prikazuje kratku analizu nekoliko priča odabranih iz zbirke priča koje se temelje na stvarnim događajima, pod naslovom Buio (Tama), spisateljice Dacie Maraini, objavljene 1999. godine u izdanju Rizzoli. Zbirka je u istoj godini osvojila nagradu Strega. U ovoj će se analizi pozornost posvetiti temama koje propituje autorica, kao što je slučaj nasilja nad djecom. Perspektiva koju nam nudi ovo djelo prožeta je silovanom djecom, prodanom i ubijenom, okruženom dubokom ravnodušnošću društva naspram toga razornog nasilja. Stoga se kroz priče Dacie Maraini želio elaborirati put koji bi vodio do njihova novoga tumačenja te naveo čitatelja na promišljanje i analizu njezina djela. Autorica obrađuje teške teme te progovara o njima kako bi na taj način promicala nadu žrtvama nasilja i zlostavljanja te da bi budućim generacijama osigurala realnost koja bi bila netolerantna na bilo koju vrstu nasilja.

Ključne riječi: Dacia Maraini, Buio (Tama), talijanska književnost, djetinjstvo, nasilje

\section{VIOLENCE IN DACIA MARAINI'S WORK DARKNESS}

This paper provides a brief analysis of selected short stories based on real events, entitled Buio (Darkness), by Dacia Maraini (published in 1999 by Rizzoli). This work won the Strega Prize in the same year. In this paper the focus is on the themes central to this book, which in this case is violence against children. The perspective this work offers us is one of children raped, killed and sold, surrounded by a society's profound indifference toward such devastating violence. A new path toward the interpretation of these stories is what is attempted here which, in turn, should lead the reader to better comprehend such disturbing content; hope for the victims of such violence and abuse is what the reader should attain, as well as to struggle to ensure that future generations will find a reality intolerant to violence of any kind.

Keywords: Dacia Maraini, Darkness, Italian literature, Childhood, Violence 